\title{
Novel tau filament fold in corticobasal degeneration, a four-repeat tauopathy
}

Wenjuan Zhang ${ }^{1}$, Airi Tarutani ${ }^{2}$, Kathy L. Newell ${ }^{3}$, Alexey G. Murzin ${ }^{1}$, Tomoyasu Matsubara ${ }^{4}$, Benjamin Falcon ${ }^{1}$, Ruben Vidal ${ }^{3}$, Holly $\mathrm{J}$. Garringer ${ }^{3}$, Yang $\mathrm{Shi}^{1}$, Takeshi Ikeuchi ${ }^{5}$, Shigeo Murayama ${ }^{4}$, Bernardino Ghetti ${ }^{3}$, Masato Hasegawa ${ }^{2}$, Michel Goedert ${ }^{1 *} \&$ Sjors H.W. Scheres ${ }^{1 *}$

${ }^{1}$ MRC Laboratory of Molecular Biology, Cambridge, $\mathrm{CB} 2 \mathrm{OQH}$, United Kingdom

${ }^{2}$ Department of Dementia and Higher Brain Function, Tokyo Metropolitan Institute of Medical Science, Tokyo, 156-8506, Japan.

${ }^{3}$ Department of Pathology and Laboratory Medicine, Indiana University School of Medicine, Indianapolis, IN 46202, USA

${ }^{4}$ Department of Neuropathology, Tokyo Metropolitan Institute of Gerontology, Tokyo, 173-0015, Japan.

${ }^{5}$ Department of Molecular Genetics, Brain Research Institute, Niigata University, Niigata 951-8585, Japan

* These authors jointly supervised this work. e-mail: mg@mrc-Imb.cam.ac.uk; scheres@mrc-Imb.cam.ac.uk 
Corticobasal degeneration (CBD) is a neurodegenerative tauopathy that is characterised by motor and cognitive disturbances (1-3). A higher frequency of the $H 1$ haplotype of MAPT, the tau gene, is present in cases of CBD than in controls $(4,5)$ and genome-wide association studies have identified additional risk factors (6). By histology, astrocytic plaques are diagnostic of $\mathrm{CBD}(7,8)$, as are detergentinsoluble tau fragments of $37 \mathrm{kDa}$ by SDS-PAGE (9). Like progressive supranuclear palsy (PSP), globular glial tauopathy (GGT) and argyrophilic grain disease (AGD) (10), CBD is characterised by abundant filamentous tau inclusions that are made of isoforms with four microtubule-binding repeats (4R) (11-15). This distinguishes $4 R$ tauopathies from Pick's disease, filaments of which are made of threerepeat (3R) tau isoforms, and from Alzheimer's disease and chronic traumatic encephalopathy (CTE), where both 3R and 4R tau isoforms are found in the filaments (16). Here we report the structures of tau filaments extracted from the brains of three individuals with CBD using electron cryomicroscopy (cryo-EM). They were identical between cases, but distinct from those of Alzheimer's disease, Pick's disease and CTE (17-19). The core of CBD filaments comprises residues K274-E380 of tau, spanning the last residue of $R 1$, the whole of $R 2$, $R 3$ and $R 4$, as well as 12 amino acids after 
R4. It adopts a novel four-layered fold, which encloses a large non-proteinaceous density. The latter is surrounded by the side chains of lysine residues 290 and 294 from $R 2$ and 370 from the sequence after R4. CBD is the first $4 R$ tauopathy with filaments of known structure.

We extracted tau filaments from the frontal cortex of three individuals with a neuropathologically confirmed diagnosis of CBD. Abundant neuronal inclusions and astrocytic plaques were stained by antibodies specific for 4R tau (Figure 1a-c) and for hyperphosphorylated tau (Figure 1e), as well as by Gallyas-Braak silver (Figure 1f). Antibodies against 3R tau failed to give specific staining (Figure 1d). By immunoblotting of sarkosyl-insoluble fractions, two major tau bands of 64 and $68 \mathrm{kDa}$ were stained by an antibody specific for $4 \mathrm{R}$ tau, as were two minor bands of $37 \mathrm{kDa}$ (Figure 1g). Immunogold negative-stain EM with antibodies specific for the $\mathrm{N}$ - and C-termini of tau, as well as for R1, R2, R3 and R4, indicated that epitopes of R2, R3 and R4 form part of the ordered cores of filaments in all three cases of CBD (Extended Data Figure 1). This is consistent with the estimated lengths of trypsin-resistant cores of CBD filaments (20). Narrow and wide filaments were present (Figure 1h), in agreement with previous findings (21). Narrow filaments have a helical twist with a crossover distance of approximately $900 \AA$ and a minimal width of $80 \AA$, a maximal width of $130 \AA$. Wide filaments have a crossover distance of 
approximately $1,300 \AA$ and a maximal width of $260 \AA$ with similar minimal width as narrow ones. We named these filaments Type I (narrow) and Type II (wide) CBD filaments, respectively. The ratios of Type II to Type I filaments ranged from 3:1 to 1:1, depending on cases. Co-pathologies are often found in CBD $(22,23)$. Small amounts of assembled TDP-43 were present in frontal cortex of CBD cases 1 and 2; CBD case 3 was negative (Extended Data Figure 2). It has been reported that $C 90 r f 72$ intermediate repeat expansions are associated with a subset of cases of CBD (24). Such expansions were not present in CBD cases 1-3. CBD case 1 had 2 repeats on each allele, CBD case 2 also had 2 repeats on each allele and CBD case 3 had 2 repeats on one allele and 11 repeats on the other. Staining for $A \beta$ in frontal cortex was stage $A$ for $C B D$ case 1 and stage 0 for CBD cases 2 and 3. Abundant $\alpha$-synuclein inclusions were not present in CBD cases 1-3, nor were inclusions positive for FUS or positive for dipeptide repeats.

We used cryo-EM and helical reconstruction in RELION (25) to determine the structures of both types of tau filaments of CBD (Figure 2a, Extended Data Table 1). In the three cases, Type I filaments are composed of a single protofilament and adopt a novel four-layered fold. Like CTE filaments, each protofilament of CBD contains an additional density that is surrounded by density of the tau protein chain. Unlike CTE (19), the additional density is found in a positively charged environment. Type II filaments consist of pairs 
of identical protofilaments of Type I, related by C2 symmetry, with less well-resolved maps at the ends of the cores than in their central parts. For case 1, we obtained maps of Type I and Type II tau filaments at overall resolutions of $3.2 \AA$ and $3.0 \AA$, respectively (Extended Data Figure 3a-d). Local resolution in the central part of Type II filaments extended to $2.8 \AA$. The maps showed side chain densities and $\beta$-strands that were well separated along the helical axis, allowing us to generate stereochemically refined atomic models (Figure 2b, Extended Data Figure 3e-h).

Type I and Type II tau filaments contain a common protofilament, whose core structure (CBD fold) is composed of residues K274-E380, i.e. the last residue of $\mathrm{R} 1$, all of R2-R4, and 12 amino acids after $\mathrm{R} 4$. In the core, there are eleven $\beta$-strands $(\beta 1-\beta 11)$ : three from R2 ( $\beta 1$ $\beta 3)$, three from R3 ( $\beta 4-\beta 6)$, four from R4 ( $\beta 7-\beta 10)$ and one from the sequence after R4 ( $\beta 11)$. They are connected by turns and arcs and form a four-layered structure (Figure 2b, Extended Data Figure 4). The central four layers are formed by $\beta 7, \beta 4, \beta 3$ and $\beta 10$. Strands $\beta 3$ and $\beta 4$ are connected by a sharp turn, whereas $\beta 7$ and $\beta 10$ are connected through $\beta 8$ and $\beta 9$, which wrap around the turn. On the other side, $\beta 2, \beta 5$ and $\beta 6$ form a three-layered structure. $\beta 2$ packs against one end of $\beta 5$ and $\beta 6$ packs against the other end. The first and the last strands, $\beta 1$ and $\beta 11$, pack against each other and close a hydrophilic cavity formed by residues from $\beta 2, \beta 3, \beta 10, \beta 11$ and 
the connections between $\beta 1$ and $\beta 2$, as well as between $\beta 2$ and $\beta 3$

(Figure 2b, Extended Data Figure 4). All interfaces in the CBD fold have mixed compositions of polar and hydrophobic groups (Extended Data Figure 4a).

Each tau repeat contains a PGGG motif (16). In the CBD fold, the PGGG motif of R2 (residues 301 to 304) forms a tight turn between $\beta 3$ and $\beta 4$, which is essential for the formation of the four-layered cross- $\beta$ packing. The PGGG motif of R3 (residues 332 to 335 ) adopts an extended conformation between $\beta 6$ and $\beta 7$, compensating for the shorter lengths of these strands compared to the opposing $\beta 4$ and $\beta 5$ connected by P312. The PGGG motif of R4 (residues 364367) adopts a similar extended conformation, forming part of the hydrophilic cavity.

In CBD Type II tau filaments, the two protofilaments are related by C2 symmetry (Figure 2b, Extended Data Figure 4). The interface between protofilaments is formed by anti-parallel stacking of ${ }^{343}$ KLDFKDR ${ }^{349}$. Besides van der Waals interactions between the anti-parallel side chains of K347 from each protofilament, the $\varepsilon^{-}$ amino group of $\mathrm{K} 347$ forms hydrogen bonds with the carboxyl group of D348 and the backbone carbonyl of K347 on the opposite protofilament (Figure 2b, Extended data Figure 5).

The hydrophilic cavity contains an additional density that is different from tau (Figure 2b, Extended Data Figure 4). The 
positively charged side chains of K290, K294 and K370 point to this extra density, which is not connected to the density for tau, indicating that it is not covalently linked to tau, and therefore cannot represent a post-translational modification. The extra density is as strong as that of tau, suggesting near-stoichiometric occupancy (Figure 2a). In cross-section, the density has approximate dimensions of $9 \AA$ by $4 \AA$, while it has only few features along the helical axis. Attempts to resolve additional features by performing refinements with a larger helical rise failed. We hypothesise that the extra density is made of non-proteinaceous, polyanionic molecules with a charge of -3 per rung. The buried nature of the negatively charged molecules, their high occupancy and presence in most filaments at end-stage disease indicate that they are continuously incorporated during filament formation. It is therefore possible that these molecules stabilise the CBD fold during initial filament assembly and/or subsequent seeded aggregation. Candidate molecules include cellular metabolites with phosphate and/or carboxylic groups, such as polyphosphate and phosphoglycerate.

CBD is characterised by abundant neuronal and glial inclusions of 4R tau. It has been suggested that tau inclusions may form first in astrocytes of striatum and prefrontal cortex (26). Astrocytic plaques, in which assembled $4 \mathrm{R}$ tau is present in the end-feet of astrocytes (6), have been reported to have a perivascular localisation (27), 
suggesting that in CBD cofactors for tau assembly may enter the brain from the periphery, similar to what we have hypothesised for CTE (19).

Six tau isoforms are expressed in adult human brain, three isoforms with $3 R$ and three isoforms with $4 R(28)$. Based on the tau isoform composition of their filaments, three different types of tauopathies can be distinguished: $3 R, 4 R$ and $3 R+4 R$. We previously reported the structures of tau filaments from Alzheimer's disease, Pick's disease and CTE (Figure 3) (17-19). Pick's disease is a 3R tauopathy, whereas both Alzheimer's disease and CTE are 3R+4R tauopathies. Tau filaments from Alzheimer's disease and CTE are different, indicating that tau isoform composition is not the sole determinant of conformation. CBD is the first example of a $4 R$ tauopathy of known filament structure. Differences in protofilament structure are observed between diseases, but not between subjects with a given disease, consistent with the existence of distinct conformers of assembled tau in different tauopathies (Figure 3).

The cores of tau filaments from human brain of known structure all contain R3, R4 and 10-12 amino acids after R4 (17-19). Filaments comprising 3R+4R tau in Alzheimer's disease and CTE do not have R1 or R2 in their cores, and those comprising 3R tau in Pick's disease have part of R1, whereas filaments comprising $4 R$ tau in $\mathrm{CBD}$ contain the whole of R2. This makes the CBD fold the largest 
known tau fold, with 107 ordered residues. Therefore, filament disassembly may come at a relatively high energetic cost, which may in turn have implications for seeded aggregation and disease progression. Tau assemblies from CBD brains have been shown to seed specific aggregation (29-31). It is likely that filaments from other 4R tauopathies, such as PSP, GGT and AGD, have also at least part of R2 in their cores, but these structures remain to be determined.

It was previously not known why only $4 \mathrm{R}$ tau isoforms are present in the filaments of CBD. Our structure reveals that S305, the last residue of $\mathrm{R} 2$, which starts $\beta 4$, is located at a position, where the side chain of $\mathrm{K} 274$, the last residue of $\mathrm{R} 1$, cannot fit (Figure 2b). Moreover, if R1 were incorporated instead of R2, K294 would be replaced by T263, which would weaken the interaction with the extra density (Figure $2 b, d$ ). In support, the sarkosylinsoluble fraction from CBD cases 1-3, which was used for cryo-EM, seeded aggregation of soluble tau in SH-SY5Y cells expressing fulllength $4 R$, but not 3R, human tau (Extended Data Fig. 6). Similarly, CBD-tau recruited only soluble $4 R$ tau into insoluble aggregates in primary neurons, whereas Alzheimer's disease-tau recruited both $3 R$ and $4 R$ tau (31). In contrast, tau filaments extracted from the brain of a patient with Pick's disease seeded aggregation of $3 R$, but not $4 R$, human tau (18). Templated misfolding of this type may explain why only $4 R$ tau is incorporated into CBD filaments. 
Despite differences between folds, with the structures of tau filaments from four human tauopathies now known, common patterns are beginning to emerge (Figure 3). Packing between $\beta 1$ and $\beta 11$ in the CBD fold resembles that between $\beta 1$ and $\beta 8$ in the Alzheimer and CTE folds $(18,20)$. In CBD, ${ }^{274} \mathrm{KVQIINK}^{280}$ packs against ${ }^{374} \mathrm{HKLTFRE}^{380}$, whereas in Alzheimer's disease and CTE ${ }^{305} \mathrm{KVQIVYK}^{311}$ packs against ${ }^{374} \mathrm{HKLTFRE}^{380}$. Hexapeptides VQIINK and VQIVYK are necessary for cofactor-induced assembly of recombinant tau $(32,33)$, seeded aggregation in cultured cells (34) and assembly of mutant human tau in transgenic mice (35). Residues ${ }^{374} \mathrm{HKLTFRE}^{380}$ are missing from the widely used tau constructs K18 and K19, which end at E372 (36). The hairpin-like structure of $\beta 4-\beta 7$ in the CBD fold resembles that of $\beta 3-\beta 6$ in the Pick fold, with the exception of C322, which points inwards to form the sharp turn in the CBD fold, and which points outwards in the Pick fold. Interestingly, in all four tau filament folds from human brain, $\beta$-strands are formed by approximately the same residues (Figure 3); this is also true of tau filaments assembled in vitro using heparin (37). It suggests a model for the diversity of tau folds, where $\beta$-strands form fixed building blocks and the loops and turns between strands provide diversity. Tau repeats contain many glycine and proline residues, all of which are located in loops and turns. 
This work may shed light on why tau folds differ between diseases, which may in turn reveal mechanisms that lead to ordered assembly. Post-translational modifications may be important. Thus, deamidation of N279 in R2 of tau takes place in Alzheimer's disease, but not in CBD or PSP (38). This residue, which is located in $\beta 1$ of CBD, is outside the structured core of $A D$ and CTE filaments. Association of non-proteinaceous cofactors with tau filaments from human brain was unexpected, even though it is well established that such factors can induce assembly of soluble, unmodified tau protein in vitro (16). In the same way that hydrophobic molecules inside the $\beta$-helix may shape the CTE fold (19), polyanionic molecules inside the positively charged cavity may help to form the CBD fold. We previously speculated that the extra densities near K317 and K321 of tau on the periphery of the Alzheimer fold (17), which are also observed in the CTE fold (19), may be formed by ${ }^{7}$ EFE $^{9}$ of tau. They are believed to be essential for the formation of the straight tau filaments of Alzheimer's disease. Their similarity to the extra density in the CBD fold raises the possibility that nonproteinaceous molecules may also play a role in providing specificity for tau assembly into Alzheimer and CTE folds.

Determination of the CBD fold opens $4 R$ tauopathies to structural analysis. It supports the hypothesis that distinct conformers of filamentous tau define different tauopathies. We previously showed that tau filaments from Alzheimer's disease, Pick's disease and CTE 

available under aCC-BY 4.0 International license.

adopt different folds (17-19). Future investigations into what drives

the specificity of tau conformers in tauopathies may lead to novel

therapeutic opportunities. 


\section{REFERENCES}

1. Lhermitte, J., Lévy, G. \& Kyriaco, N. Les perturbations de la représentation spatiale chez les apraxiques. Rev. Neurol. (Paris) 2, 586-600 (1925).

2. Rebeiz, J.J., Kolodny, E.H. \& Richardson, E.P. Corticodentatonigral degeneration with neuronal achromasia. Arch. Neurol. 18, 20-33 (1968).

3. Gibb, W.R.G., Luthert, P.J. \& Marsden, C.D. Corticobasal degeneration. Brain 112, 1171-1192 (1989).

4. Di Maria, E. et al. Corticobasal degeneration shares a common genetic background with progressive supranuclear palsy. Ann. Neurol. 47, 374-377 (2000).

5. Houlden, $\mathrm{H}$. et al. Corticobasal degeneration and progressive supranuclear palsy share a common tau haplotype.

Neurology 56, 1702-1706 (2001).

6. Kouri, N. et al. Genome-wide association study of corticobasal degeneration identifies risk variants shared with progressive supranuclear palsy. Nat. Commun. 6, 7247 (2015).

7. Feany, M.B. \& Dickson, D.W. Widespread cytoskeletal pathology characterizes corticobasal degeneration. Am. J. Pathol. 146, 1388-1396 (1995).

8. Komori, T. et al. Astrocytic plaques and tufts of abnormal fibers do not coexist in corticobasal degeneration and 
progressive supranuclear palsy. Acta Neuropathol. 96, 401407 (1998).

9. Arai, T. et al. Identification of amino-terminally cleaved tau fragments that distinguish progressive supranuclear palsy from corticobasal degeneration. Ann. Neurol. 55, 72-79 (2004).

10. Rösler, T.W. et al. Four-repeat tauopathies. Prog. Neurobiol. 180,101644 (2019).

11. Paulus, W. \& Selim, W. Corticonigral degeneration with neuronal achromasia and basal neurofibrillary tangles. Acta Neuropathol. 81, 89-94 (1990).

12. Wakabayashi, K. et al. Corticobasal degeneration: Etiopathological significance of the cytoskeletal alterations. Acta Neuropathol. 87, 545-553 (1994).

13. Ksiezak-Reding, $\mathrm{H}$. et al. Ultrastructure and biochemical composition of paired helical filaments in corticobasal degeneration. Am. J. Pathol. 145, 1496-1508 (1994).

14. Arima, K. et al. Corticonigral degeneration with neuronal achromasia presenting with primary progressive aphasia: Ultrastructural and immunocytochemical studies. J. Neurol. Sci. 127, 186-197 (1994).

15. Sergeant, N., Wattez, A. \& Delacourte, A. Neurofibrillary degeneration in progressive supranuclear palsy and 
corticobasal degeneration: Tau pathologies with exclusively

"exon 10" isoforms. J. Neurochem. 72, 1243-1249 (1999).

16. Goedert, M., Eisenberg, D.S. \& Crowther, R.A. Propagation of tau aggregates and neurodegeneration. Annu. Rev. Neurosci. 40, 189-210 (2017).

17. Fitzpatrick, A.W.P. et al. Cryo-EM structures of tau filaments from Alzheimer's disease. Nature 547, 185-190 (2017).

18. Falcon, B. et al. Structures of filaments from Pick's disease reveal a novel tau protein fold. Nature 561, 137-140 (2018).

19. Falcon, B. et al. Novel tau filament fold in chronic traumatic encephalopathy encloses hydrophobic molecules. Nature 568, 420-423 (2019).

20. Taniguchi-Watanabe, S. et al. Biochemical classification of tauopathies by immunoblot, protein sequence and mass spectrometric analyses of sarkosyl-insoluble and trypsinresistant tau. Acta Neuropathol. 131, 267-280 (2016).

21. Ksiezak-Reding, H. et al. Ultrastructural instability of paired helical filaments from corticobasal degeneration as examined by scanning transmission electron microscopy. Am. J. Pathol. $149,639-651$ (1996).

22. Robinson, J.L. et al. Neurodegenerative disease concomitant proteinopathies are prevalent, age-related and APOE4associated. Brain 141, 2181-2193 (2018). 
23. Uryu, K. et al. Concomitant TAR-DNA-binding protein 43 pathology is present in Alzheimer disease and corticobasal degeneration but not in other tauopathies. J. Neuropathol. Exp. Neurol. 67, 555-564 (2008).

24. Cali, C.P. et al. C9orf72 intermediate repeats are associated with corticobasal degeneration, increased C90rf72 expression and disruption of autophagy. Acta Neuropathol., in press.

25. He, S. \& Scheres, S.H.W. Helical reconstruction in RELION. J. Struct. Biol. 198, 163-176 (2017).

26. Ling, $\mathrm{H}$. et al. Astrogliopathy predominates the earliest stage of corticobasal degeneration pathology. Brain 139, 32373252 (2016).

27. Shibuya, K. et al. Perivascular orientation of astrocytic plaques and tuft-shaped astrocytes. Brain Res. 1404, 50-54 (2011).

28. Goedert, M. et al. Multiple isoforms of human microtubuleassociated protein tau: Sequences and localization in neurofibrillary tangles of Alzheimer's disease. Neuron 3, 519526 (1989).

29. Clavaguera, F. et al. Brain homogenates from human tauopathies induce tau inclusions in mouse brain. Proc. Natl. Acad. Sci. USA 110, 9535-9540 (2013). 
30. Sanders, D.W. et al. Distinct tau prion strains propagate in cells and mice and define different tauopathies. Neuron 82, 1271-1288 (2014).

31. Narasimhan, S. et al. Pathological tau strains from human brains recapitulate the diversity of tauopathies in nontransgenic mouse brain. J. Neurosci. 37, 11406-11423 (2017).

32. Von Bergen, M. et al. Assembly of t protein into Alzheimer paired helical filaments depends on a local sequence motif (306VQIVYK311) forming $\beta$ structure. Proc. Natl. Acad. Sci. USA 97, 5129-5134 (2000).

33. Von Bergen, M. et al. Mutations of tau protein in frontotemporal dementia promote aggregation of paired helical filaments by enhancing local $\beta$-structure. J. Biol. Chem. 276, 48165-48174 (2001).

34. Falcon, B. et al. Conformation determines the seeding potencies of native and recombinant tau aggregates. J. Biol. Chem. 290, 1049-1065 (2015).

35. Macdonald, J.A. et al. Assembly of transgenic human P301S Tau is necessary for neurodegeneration in murine spinal cord. Acta Neuropathol. Commun. 7, 44 (2019).

36. Gustke, N. et al. Domains of tau protein and interactions with microtubules. Biochemistry 33, 9511-9522 (1994). 
37. Zhang, W. et al. Heparin-induced tau filaments are polymorphic and differ from those in Alzheimer's and Pick's diseases. elife 8: e43584 (2019).

38. Dan, A. et al. Extensive deamidation at asparagine residue 279 accounts for weak immunoreactivity of tau with RD4 antibody in Alzheimer's disease brain. Acta Neuropathol. Commun. 1: 54 (2013). 


\section{FIGURE LEGENDS}

\section{Figure 1.}

\section{Filamentous tau pathology of CBD.}

(a-f), Staining of neuronal inclusions, neuropil threads and astrocytic plaques in the frontal cortex of CBD cases 1-3 by antibody RD4 (specific for 4R tau, brown) (a-c), and in the frontal cortex of case 3 by antibody AT8 (pS202, pT205 tau, brown) (e) and Gallyas-Braak silver (black) (f). Staining of frontal cortex from CBD cases 1-3 was negative when antibody RD3 (specific for 3R tau) was used (d). Nuclei were counterstained in blue. Scale bars, $50 \mu \mathrm{m}$. (g), Immunoblots using antibodies RD4, RD3 and AT8 of sarkosyl-insoluble tau extracted from the frontal cortex of CBD cases 1-3. (h), Negative-stain electron micrographs of Type I (narrow) and Type II (wide) tau filaments extracted from the frontal cortex of CBD case 1 . Scale bar, $50 \mathrm{~nm}$.

\section{Figure 2.}

\section{Cryo-EM maps of CBD Type I and Type II tau filaments and atomic model of Type II filaments.}

(a), Cryo-EM maps of Type I tau filaments (upper panels) and Type II tau filaments (lower panels) from the frontal cortex of cases 1-3. 
(b), Atomic model of the CBD Type II tau filament (upper panel).

The extra density is shown in light blue, with K290, K294 and K370 indicated. Schematic depicting the microtubule-binding repeats (R1R4) of tau and the sequence after R4 that is present in the core of CBD filaments (all shown in different colours) (lower panel). The positions of $\beta$-strands $(\beta 1-\beta 11)$ are indicated.

\section{Figure 3.}

\section{Structures of tau filament cores from human brain.}

(a), Protofilament from corticobasal degeneration (CBD fold), a $4 R$ tauopathy; protofilament from Pick's disease (Pick fold), a 3R tauopathy; protofilaments from Alzheimer's disease (Alzheimer fold) and chronic traumatic encephalopathy (CTE fold), both $3 R+4 R$ tauopathies. Red arrows point to the internal, non-proteinaceous densities in CBD and CTE folds. (b), Schematic depicting the microtubule binding repeats (R1-R4) of tau and the sequence after R4, with the $\beta$-strands found in the cores of tau filaments in the different diseases marked by thick arrows. 
Acknowledgements We thank the patients' families for donating brain tissues; F. Epperson, R.M. Richardson and U. Kuederli for brain collection and technical support with neuropathology; G. Cannone, S. Chen, J. Brown, G. Sharov, and A. Yeates for support with electron microscopy; T. Nakane for help with RELION; G. Murshudov and R. Warshamanage for help with REFMAC; P. Emsley for help with COOT; T. Darling and J. Grimmett for help with high-performance computing; R.A. Crowther for helpful discussions. W. Z. was supported by a Foundation that prefers to remain anonymous. M.G. is an Honorary Professor in the Department of Clinical Neurosciences of the University of Cambridge. This work was supported by the U.K. Medical Research Council (MC_U105184291 to M.G. and MC_UP_A025_1013 to S.H.W.S.), the European Union (Joint Programme-Neurodegeneration Research REfrAME, to B.F. and M.G., and the EU/EFPIA/Innovative Medicines Initiative [2] Joint Undertaking IMPRiND, project 116060, to M.G.), the Japan Agency for Science and Technology (Crest, JPMJCR18H3), to M.H., the Japan Agency for Medical Research and Development (JP18ek0109391 and JP18dm020719), to M.H. and (JP19ek0109392), to T.I., the U.S. National Institutes of Health (P30AG010133 and UO1NS110437), to R.V. and B.G., and the Department of Pathology and Laboratory Medicine, Indiana University School of Medicine, to R.V. and B.G. This study was supported by the MRC-LMB EM facility. We acknowledge the Center 
for Medical Genomics of Indiana University School of Medicine for next-generation DNA sequencing.

Author contributions A.T., K.L.N., T.M., S.M., B.G. and M.H. identified patients, performed neuropathology and extracted tau filaments from CBD cases 1 and 2; R.V., H.J.G. and T.I. carried out genetic analyses; W.Z. extracted tau filaments from CBD case 3 and conducted immunolabelling. W. Z. and B. F. performed cryo-EM; W.Z., Y.S. and S.H.W.S. analysed the cryo-EM data; W.Z. and A.G.M. built the atomic models; A.T. and M.H. carried out seeded aggregation; M.G. and S.H.W.S. supervised the project; all authors contributed to writing the manuscript. 


\section{METHODS}

Clinical history and neuropathology. Frontal cortex from three patients with a neuropathologically confirmed diagnosis of CBD was used. Case 1 was a female from Japan who died aged 74 following a six year history of progressive memory loss and motor impairment. Case 2 was a female from Japan who died aged 79 following a nine year history of progressive memory impairment and spatial disorientation. Case 3 was a male from the U.S. who died aged 52 following a seven year history of personality changes and cognitive dysfunction. Neuropathologically, the brains of all three cases exhibited signs of $\mathrm{CBD}$, with abundant $4 \mathrm{R}$ tau- and silverpositive neuronal inclusions, neuropil threads and astrocytic plaques (Figure 1a-e). Tufted astrocytes characteristic of progressive supranuclear palsy (PSP) were not observed. By immunoblotting of the sarkosyl-insoluble fraction, bands of 64 and $68 \mathrm{kDa}$ were detected by antibodies RD4 and AT8, but not by RD3. Bands of 37 kDa were also seen, consistent with CBD (Figure $1 \mathrm{~g}$ ). No known disease-causing mutations were present in MAPT. All three cases were homozygous for $A$ at position 152; A152T tau has been reported to be a risk factor for some tauopathies $(39,40)$. Whole- 
exome and whole-genome sequencing did not detect mutations known to cause Alzheimer's disease, Parkinson's disease, frontotemporal dementia or amyotrophic lateral sclerosis. All three cases were homozygous for the $H 1$ haplotype of MAPT. Two haplotypes, $H 1$ and $H 2$, are present in the Caucasian population, with the $H 1$ haplotype being over-represented in individuals with CBD and PSP $(4,5)$. The Japanese population has only $H 1(41,42)$. The APOE genotypes of CBD cases $1-3$ were: case $1(\varepsilon 3 / \varepsilon 4)$, case 2 $(\varepsilon 3 / \varepsilon 3)$, case $3(\varepsilon 3 / \varepsilon 3)$.

Whole-exome sequencing. Target enrichment made use of the SureSelectTX human all-exon library (V6, $58 \mathrm{Mb}$, Agilent) and high-throughput sequencing was carried out using a HiSeq4,000 (2x75-bp paired-end configuration, Illumina). Bioinformatics analyses were performed as described (43).

Whole-genome sequencing. Sequencing libraries were prepared using $100 \mathrm{ng}$ high-quality genomic DNA from cerebellum using Illumina Nextera DNA Flex Library Prep Kit, and assessed using a Qubit and Agilent Bioanalyzer. High-throughput DNA sequencing was carried out on multiple libraries pooled in equal molarity using a NovaSeq6,000 (150-bp paired-end configuration, Illumina) and aligned to the human reference genome GRCh38 using BWA and Bwakit (v.0.7.15). ExpansionHunter was applied to estimate expansion numbers of short tandem repeats (v.2.5.5) and 
germline variants were identified with strelka2 (v.2.9.9), with default parameters for whole-genome sequence data. The variants were annotated for their effects with ANNOVAR (44).

C9orf72 hexanucleotide repeat expansion. Repeat-primed polymerase chain reaction was used to determine the number of GGGGCC hexanucleotide repeats in the first intron of $C 90 r f 72$ (Asuragen AmplideX PCR/CE C9orf72 kit). Internal standards were analysed along with samples to evaluate assay performance. Repeat numbers of up to 25 repeats were determined with an accuracy of \pm 1 repeat, and repeat numbers greater than 25 were determined with an accuracy of \pm 3 repeats.

Extraction of tau filaments. Sarkosyl-insoluble material was extracted from fresh-frozen frontal cortex of CBD cases 1-3, essentially as described (20). Briefly, tissues were homogenised in 20 volumes ( $/ \mathrm{v})$ extraction buffer consisting of $10 \mathrm{mM}$ Tris- $\mathrm{HCl}, \mathrm{pH}$ 7.5, $0.8 \mathrm{M} \mathrm{NaCl}, 10 \%$ sucrose and $1 \mathrm{mM}$ EGTA. Homogenates were brought to $2 \%$ sarkosyl and incubated for $30 \mathrm{~min}$. at $37^{\circ} \mathrm{C}$.

Following a $10 \mathrm{~min}$. centrifugation at $20,000 \mathrm{~g}$, the supernatants were spun at $100,000 \mathrm{~g}$ for $20 \mathrm{~min}$. The pellets were resuspended in $700 \mu \mathrm{l} / \mathrm{g}$ extraction buffer and centrifuged at $9,500 \mathrm{~g}$ for $10 \mathrm{~min}$. For $\mathrm{CBD}$ cases 1 and 2, the supernatants were diluted 3 -fold in 50 $\mathrm{mM}$ Tris- $\mathrm{HCl}, \mathrm{pH} 7.5$, containing $0.15 \mathrm{M} \mathrm{NaCl}, 10 \%$ sucrose and $0.2 \%$ sarkosyl and spun at $166,000 \mathrm{~g}$ for $30 \mathrm{~min}$. For CBD case 3, 
the supernatant was spun at $100,000 \mathrm{~g}$ for $60 \mathrm{~min}$ and the pellet resuspended in $700 \mu \mathrm{l} / \mathrm{g}$ extraction buffer and centrifuged at 9,800 g. The supernatant was then spun at $100,000 \mathrm{~g}$ for $60 \mathrm{~min}$. Sarkosyl-insoluble pellets of CBD cases 1-3 were resuspended in 25 $\mu \mathrm{l} / \mathrm{g}$ of $20 \mathrm{mM}$ Tris- $\mathrm{HCl}, \mathrm{pH} 7.4,100 \mathrm{mM} \mathrm{NaCl}$ and used for cryo-EM. For immuno-EM the samples were diluted 5-10-fold. Sarkosylinsoluble pellets of approximately $2 \mathrm{~g}$ frontal cortex were used for cryo-EM.

\section{Immunolabelling, histology and silver staining.}

Western blotting and immunogold negative-stain EM were carried out as described (45). For Western blotting, the samples were resolved on $4-20 \%$ Tris-glycine gels (Novex) and the primary antibodies were diluted in PBS plus $0.2 \%$ Tween-20, $1 \%$ bovine serum albumin. Primary antibodies were: RD3 and RD4 (46) (Millipore), used at 1:4,000; AT8 (47) (specific for pS202/pT205 tau) (Thermo Fisher), used at 1:1,000 and anti-phospho-TDP-43 (pS409/pS410) $(48,49)$ (Cosmo Bio), used at 1:1,000. For immunoEM, primary antibodies were used at 1:50. They were: BR133 (28) (raised against tau residues 1-16), BR136 (18) (raised against tau residues 244-257), Anti-4R $(38,50)$ (raised against tau residues 275-291), BR135 (28) (raised against tau residues 323-335), TauC4 (20) (raised against tau residues 354-369) and BR134 (28) (raised against tau residues 428-441). Histology and immunohistochemistry were carried out as described (51). Brain 
sections were 8 micron-thick and were counterstained with haematoxylin. Primary antibodies were: RD3 (1:1,000); RD4 $(1: 1,000) ; \operatorname{AT8}(1: 300) ;$ anti-pS396 tau $(1: 1,000$, Calbiochem); anti-TauC, specific for tau at residues $422-438$ (1:1,000, Cosmo Bio); anti-phospho-TDP-43 (1:1,000); anti-FUS (1:200, Sigma); anti-poly-GA (1:1,000, Cosmo Bio); anti- $\alpha$-tubulin $(1: 1,000$, Sigma) and anti-influenza hemagglutinin (HA) (1:1,000, Sigma). Sections were silver-impregnated using the method of Gallyas-Braak to visualise inclusions $(52,53)$.

Seeded tau aggregation. Seeded aggregation was carried out as described (54), except that sarkosyl-insoluble tau from CBD cases 1-3 was used as seed. Briefly, 10 ng sarkosyl-insoluble tau seed and Multifectam (Promega) were added to SH-SY5Y cells transiently expressing HA-tagged 1 N3R or 1N4R human tau. Mock transfections were done in the absence of tau seeds. After three days of culture, sarkosyl-insoluble and sarkosyl-soluble fractions were prepared and used for immunoblotting. Insoluble tau was detected with anti-HA and anti-pS396-tau antibodies. Total tau was detected with antiTauC. Tau concentrations in the seeds from frontal cortex of CBD cases 1-3 were determined using the Tau ELISA kit Wako (FUJIFILM). The intensity of HA-positive bands in the sarkosylinsoluble fractions was quantified using Image J software. 
Electron cryo-microscopy. Extracted tau filaments were centrifuged at 3,000 $\mathrm{g}$ for $30 \mathrm{~s}$, before being applied to glowdischarged holey carbon grids (Quantifoil Au R1.2/1.3, 300 mesh) and plunge-frozen in liquid ethane using a Thermo Fischer Vitrobot Mark IV. Images were acquired on a Gatan K2-Summit detector in counting mode using a Thermo Fischer Titan Krios microscope at 300 kV. A GIF-quantum energy filter (Gatan) was used with a slit width of $20 \mathrm{eV}$ to remove inelastically scattered electrons. Further details are given in Extended Data Table 1.

Helical reconstruction. Movie frames were gain-corrected, aligned, dose-weighted and then summed into a single micrograph using MOTIONCOR2 (54). The micrographs were used to estimate the contrast transfer function (CTF) using Gctf (55). All subsequent image-processing steps were performed using helical reconstruction methods in RELION $3.0(25,56,57)$. Both types of filaments were selected manually in the micrographs, and the resulting data sets were processed independently. For Type I (narrow) filaments, 135,646 segments were extracted with an inter-box distance of $14.1 \AA$ and a box size of 920 pixels. Initial reference-free 2D classification was performed with images that were downscaled to 230 pixels to speed up calculations. Segments contributing to suboptimal 2D class averages were discarded. Assuming a helical rise of $4.75 \AA$, a helical twist of $-0.9^{\circ}$ was estimated from the crossover distance of filaments in the micrographs. Using these 
parameters, an initial 3D reference was reconstructed from the 2D class averages de novo. We then re-extracted the selected segments without downscaling them, and with a smaller box of 330 pixels. Using these segments and the de novo initial model low-pass filtered to $15 \AA$, we carried out 3D auto-refinement. We then used the refined reconstruction, low-pass filtered to $15 \AA$, as reference for a 3D classification without further image alignment. The segments contributing to the best 3D class were used for subsequent 3D auto-refinement of 24,073 selected segments. Refinement of the helical parameters converged onto a helical twist of $-0.845^{\circ}$ and a helical rise of $4.786 \AA$. After Bayesian polishing and CTF refinement, the reconstruction was sharpened with a Bfactor of $-26.23 \AA^{2}$ (Extended Data Table 1) using the standard post-processing procedure in RELION. The overall resolution of the final map was estimated as $3.2 \AA$ from Fourier shell correlations at 0.143 between the two independently refined half-maps, using phase-randomisation to correct for convolution effects of a generous, soft-edged solvent mask that extended to $30 \%$ of the height of the box (58). Local resolution estimates were obtained using the same phase-randomisation procedure, but with a soft spherical mask that was moved over the entire map. For Type II (wide) filaments, 129,812 segments were extracted with an interbox distance of $14.1 \AA$ and a box size of 460 pixels, which were downscaled to 230 pixels for reference-free 2D classification and 
particle selection. An initial 3D reference was again reconstructed from the 2D class averages de novo by assuming a helical rise of $4.75 \AA$ and a helical twist of $-0.65^{\circ}$, based on the estimated crossover distance of filaments. The resulting reconstruction suggested the presence of two-fold symmetry in the structure. To confirm whether this symmetry also had a translational component, we performed two 3D auto-refinements, one with C2 symmetry and one with $\mathrm{C} 1$ symmetry, but imposing a pseudo- $2_{1}$ screw axis. By comparing the results of the refinements, we found that the map obtained following the application of C2 symmetry was of higher resolution. The C2 map also showed separation of $\beta$-strands along the helical axis, which was absent in the map refined with the screw symmetry. We applied C2 symmetry in all subsequent refinements. Using 3D classification without image alignment, the segments contributing to the best 3D class were selected and used for 3D auto-refinement with optimisation of helical twist and rise. A final $3 D$ auto-refinement of 20,752 selected segments in boxes of 330 pixels without downscaling converged onto a helical twist of $-0.61^{\circ}$ and the helical rise at $4.786 \AA$. Following Bayesian polishing (59) and CTF refinement, the overall resolution of the final map was estimated as 3.0 ^. For Type I and Type II tau filaments in CBD case 2 and CBD case 3, the dataset processing steps were similar to Type I and Type II tau filaments in CBD case 1 , with the initial 3D references reconstructed de novo independently for each dataset. 
Model building and refinement. The models of the cores of

Type I and Type II filaments were built de novo in combination of both $3.2 \AA$ and $3.0 \AA$ resolution reconstructions from CBD case 1 using COOT (60). We started model building from the ${ }^{301} \mathrm{PGGG}^{304}$ motif, with the aromatic side chains of H299 and Y310 not far away, and worked our way towards the $\mathrm{N}$ - and $\mathrm{C}$-terminal regions by manually adding amino acids and by targeted real-space refinement in the high-resolution core part of Type II filaments. Tracing of the chain was confirmed by the fitting of the ${ }^{332} \mathrm{PGGG}^{335}$ motif, which neighbours the side chains of H329 and H330. Since the density of side chains of N368-E380 was weak in the $3.0 \AA$ reconstruction of Type II filaments, we assigned the side chains in this region following the $3.2 \AA$ reconstruction of Type I filaments. The structure of one protofilament from Type II filaments was rigid-body fitted into the reconstruction of Type I filaments to build the model of Type I filaments. Because of the lack of interaction between two protofilaments, the conformations of the side chains of $\mathrm{K} 343$ and K347 in Type I filaments were assigned differently from their counterparts in Type II filaments, according to the reconstruction. Each model was then translated to give a stack of three consecutive monomers to preserve nearest-neighbour interactions for the middle chain in subsequent refinements. Because most residues adopted a $\beta$-strand conformation, hydrogen-bond restraints were imposed to preserve a parallel, in-register hydrogen bonding 
pattern in earlier stages of Fourier-space refinements. Local

symmetry restraints were imposed to keep all $\beta$-strand rungs

identical. Side chain clashes were detected using MOLPROBITY (61)

and corrected by iterative cycles of real-space refinement in COOT and Fourier-space refinement in REFMAC (62) and PHENIX (63). For each refined structure, separate model refinements were performed against a single half-map, and the resulting model was compared to the other half-map to confirm the absence of overfitting (Extended Data Figure $3 a, b)$. The final models were stable in refinements without additional restraints. Statistics for the final models are shown in Extended Data Table 1.

Ethical review board and informed consent. The studies carried out at Tokyo Metropolitan Institute of Medical Science and at Indiana University were approved through the ethical review process at each Institution. Informed consent was obtained from the patients' next of kin.

Data availability. Cryo-EM maps for CBD case 1 will be deposited in the Electron Microscopy Data Bank upon acceptance of manuscript for publication. Atomic models for CBD Type I and Type II tau filaments will be deposited in the Protein Data Bank upon 

available under aCC-BY 4.0 International license.

acceptance of manuscript for publication. Whole-exome and wholegenome sequencing data and repeat-primed polymerase chain reaction C9orf72 hexanucleotide repeat expansion data will be deposited in the European Genome-phenome Archive upon acceptance of manuscript for publication. 


\section{METHODS REFERENCES}

39. Kara, E. et al. The MAPT p.A152T variant is a risk factor associated with tauopathies with atypical clinical and neuropathological features. Neurobiol. Aging 33, 2231.e72231.e14 (2012).

40. Coppola, G. et al. Evidence for a role of the rare p.A152T variant in MAPT in increasing the risk for FTD-spectrum and Alzheimer's disease. Hum. Mol. Genet. 21, 3500-3512 (2012).

41. Conrad, C. et al. Differences in a dinucleotide repeat polymorphism in the tau gene between Caucasian and Japanese populations: implication for progressive supranuclear palsy. Neurosci. Lett. 250, 135-137 (1998).

42. Evans, W. et al. The tau H2 haplotype is almost exclusively Caucasian in origin. Neurosci. Lett. 369, 183-185 (2004).

43. Farlow, J.L. et al. Whole-exome sequencing in familial Parkinson disease. JAMA Neurol. 73, 68-75 (2016).

44. Wang, K., Li, M. \& Hakonarson, H. ANNOVAR: Functional annotation of genetic variants from high-throughput sequencing data. Nucleic Acids Res. 38, e164 (2010).

45. Goedert, M., Spillantini, M.G., Cairns, N.J. \& Crowther, R.A. Tau proteins of Alzheimer paired helical filaments: abnormal phosphorylation of all six brain isoforms. Neuron $8,159-168$ (1992). 
46. De Silva, R. et al. Pathological inclusion bodies in tauopathies contain distinct complements of tau with three or four microtubule-binding repeat domains as demonstrated by new specific monoclonal antibodies. Neuropathol. Appl. Neurobiol. 29, 288-302 (2003).

47. Mercken, M. et al. Monoclonal antibodies with selective specificity for Alzheimer tau are directed against phosphatase-sensitive epitopes. Acta Neuropathol. 84, 265272 (1992).

48. Hasegawa, M. et al. Phosphorylated TDP-43 in frontotemporal lobar degeneration and amyotrophic lateral sclerosis. Ann. Neurol. 64, 60-70 (2008).

49. Inukai, Y. et al. Abnormal phosphorylation of Ser $409 / 410$ of TDP-43 in FTLD-U and ALS. FEBS Lett. 582, 2899-2904 (2008).

50. Ebashi, M. et al. Detection of AD-specific four repeat tau with deamidated asparagine residue 279-specific fraction purified from 4R tau polyclonal antibody. Acta Neuropathol. 138, 163-166 (2019).

51. Spina, S. et al. The tauopathy associated with mutation +3 in intron 10 of Tau: characterization of the MSTD family. Brain 131, 72-89 (2008) 
52. Gallyas, F. Silver staining of Alzheimer's neurofibrillary changes by means of physical development. Acta Morphol. Acad. Sci. Hung. 19, 1-8 (1971).

53. Braak, H., Braak, E., Ohm,T. \& Bohl, J. Silver impregnation of Alzheimer's neurofibrillary changes counterstained for basophilic material and lipofuscin pigment. Stain Technol. 63, 197-200 (1988).

54. Nonaka, T., Watanabe, S.T., Iwatsubo, T., \& Hasegawa, M. Seeded aggregation and toxicity of alpha-synuclein and tau: cellular models of neurodegenerative diseases. J. Biol. Chem. $285,34885-34898$ (2010).

55. Zheng, S.Q. et al. MotionCor2: Anisotropic correction of beam-induced motion for improved cryo-electron microscopy. Nat. Meth. 14, 331-332 (2017).

56. Zhang, K. Gctf: Real-time CTF determination and correction. J. Struct. Biol. 193, 1-12 (2016).

57. Scheres, S.H.W. RELION: Implementation of a Bayesian approach to cryo-EM structure determination. J. Struct. Biol. $180,519-530(2012)$

58. Zivanov, J. et al. New tools for automated high-resolution cryo-EM structure determination in RELION-3. eLife 7, e42166 (2018)

59. Chen, S. et al. High-resolution noise substitution to measure overfitting and validate resolution in 3D structure 
determination by single particle electron cryomicroscopy. Ultramicroscopy 135, 24-35 (2013).

60. Zivanov, J. et al. A Bayesian approach to beam-induced motion correction in cryo-EM single-particle analysis. IUCr] 6, 5-17(2019).

61. Emsley, P., Lohkamp, B., Scott, W.G. \& Cowtan, K. Features and development of Coot. Acta Crystallogr. D 66, 486-501 (2010).

62. Chen, V.B. et al. MolProbity: all-atom structure validation for macromolecular crystallography. Acta Crystallogr. D 66, 1221 (2010).

63. Murshudov, G.N., Vagin, A.A. \& Dodson, E.J. Refinement of macromolecular structures by the maximum-likelihood method. Acta Crystallogr. D 53, 240-255 (1997).

64. Adams, P.D. et al. PHENIX: A comprehensive Python-based system for macromolecular structure solution. Acta Crystallogr. D 66, 213-221 (2010). 


\section{EXTENDED DATA FIGURE LEGENDS}

\section{Extended Data Figure 1.}

\section{Immunolabelling of tau filaments extracted from the frontal cortex of CBD cases 1-3.}

Representative immunogold negative-stain electron microscopy images of Type I and Type II tau filaments labelled with antibodies BR133, BR136 and BR134. Antibodies Anti-4R, BR135 and TauC4 did not label filaments, which indicates that their epitopes lie within the ordered filament cores. Scale bar, $50 \mathrm{~nm}$.

\section{Extended Data Figure 2.}

\section{Assembled TDP-43 in frontal cortex of CBD cases 1-3.}

a, Immunoblots using anti-phospho-TDP-43 antibody. Sarkosylinsoluble material was prepared as described and all the samples were applied on the same gel. The $43 \mathrm{kDa}$ band $(*)$ corresponds to full-length TDP-43 and the $18-26 \mathrm{kDa}$ bands (**) to C-terminal fragments. 


\section{Extended Data Figure 3.}

\section{Cryo-EM map and model comparisons.}

$\mathbf{a}, \mathbf{b}$, Fourier shell correlation (FSC) curves between two

independently refined half-maps (black, solid), of the final model versus the full map (red, solid), of a model refined in the first halfmap versus the first half-map (green, solid), and of the same model versus the second half-map (blue, dashed) for CBD Type I (a) and Type II (b) filaments. c, d, Local resolution estimates for the CBD Type I (c) and Type II (d) filament reconstructions. e, f, Side views of the 3D reconstructions of CBD Type I (c) and Type II (d) filaments. g, h, Sharpened, high-resolution cryo-EM maps of CBD Type I (g) and Type II (h) tau filaments with their corresponding atomic models overlaid.

\section{Extended Data Figure 4.}

\section{CBD tau filament fold.}

a, Schematic of the CBD fold. $\mathbf{b}$, Rendered view of the secondary structure elements in the CBD fold, depicted as three successive rungs. $\mathbf{c}$, As in $b$, but in a view perpendicular to the helical axis, revealing the changes in height within a single molecule. d, 
Comparison of the protofilament structures of CBD Type I (blue) and Type II (pink).

\section{Extended Data Figure 5.}

\section{Protofilament interface in CBD Type II tau filaments.}

Packing between residues ${ }^{343} \mathrm{KLDFKDR}^{349}$ of the two protofilaments. Inter-protofilament hydrogen bonds are shown in yellow. Intraprotofilament hydrogen bonds are shown in green.

\section{Extended Data Figure 6.}

Seeded tau aggregation induced by CBD filaments in SH-SY5Y cells.

a, Immunoblotting of sarkosyl-insoluble (Ppt) and sarkosyl-soluble (Sup.) fractions extracted from mock-transfected SH-SY5Y cells and from cells transfected with tau seeds from frontal cortex of CBD cases $1-3$. SH-SY5Y cells transiently expressed either hemagglutinin (HA)-tagged 1 N4R or HA-tagged 1 N3R human tau. Insoluble tau was detected with anti-HA and anti-pS396 tau antibodies. Total tau was detected with anti-TauC. Blotting with an anti- $\alpha$-tubulin antibody served as loading control. b, Quantitation of anti-HA- 
bioRxiv preprint doi: https://doi.org/10.1101/811703; this version posted October 21, 2019. The copyright holder for this preprint (which was not certified by peer review) is the author/funder, who has granted bioRxiv a license to display the preprint in perpetuity. It is made available under aCC-BY 4.0 International license.

positive bands. The results are expressed as the means \pm S.E.M.

$(n=3)$. 


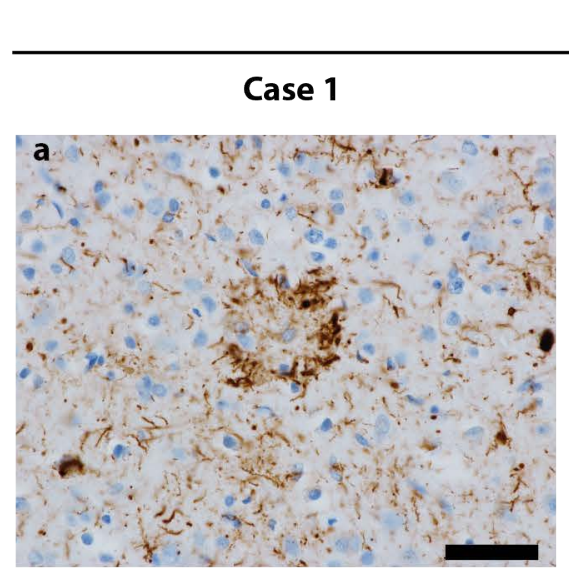

RD4 (4R tau)
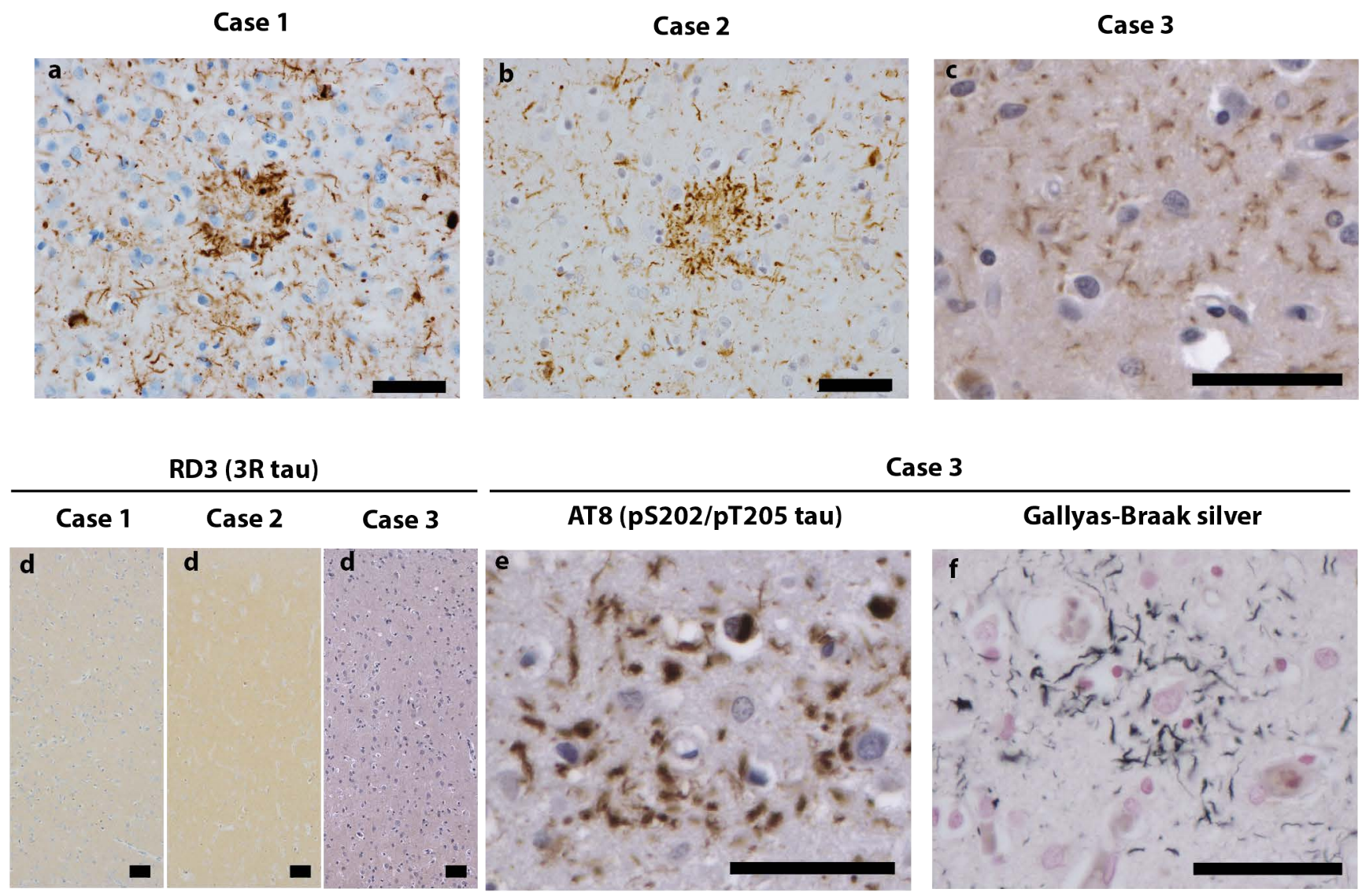

Case 3

g

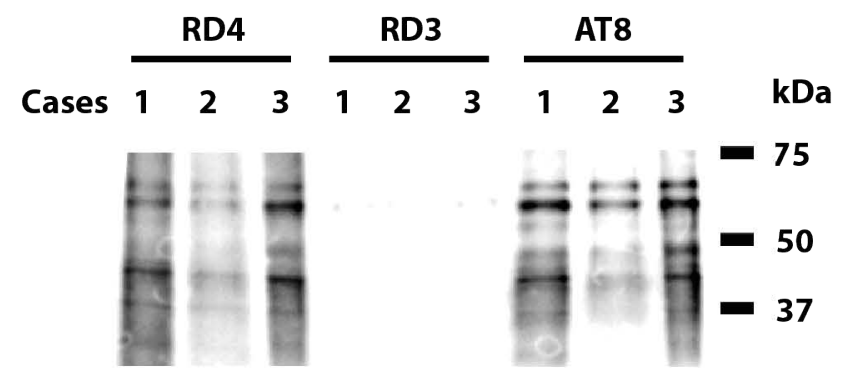

h

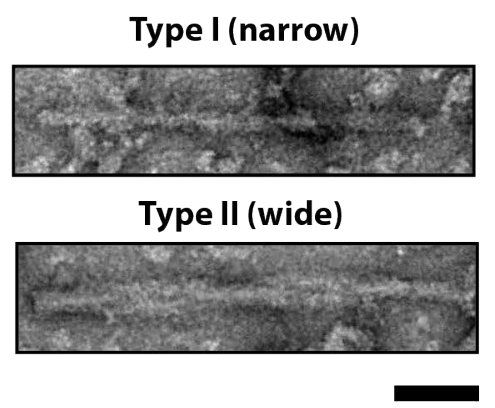


Figure 2

a

Case 1

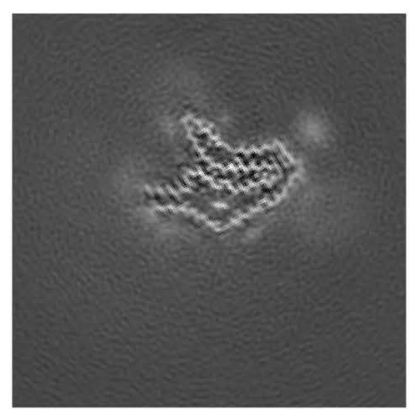

Case 1

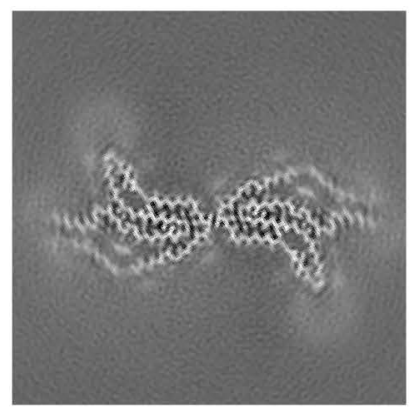

Case 2

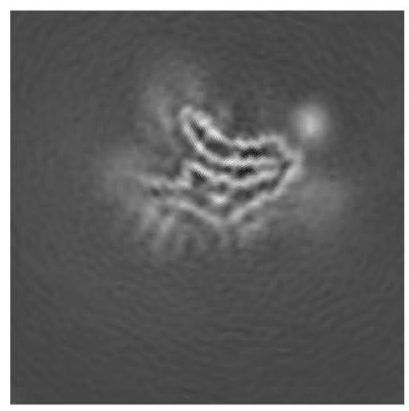

Case 2

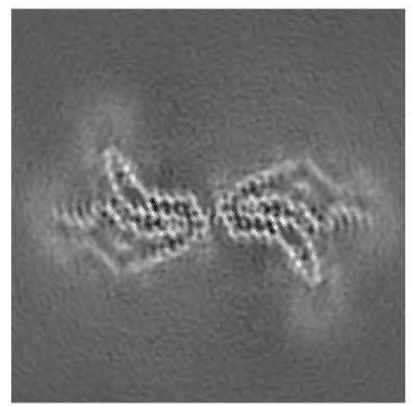

Case 3

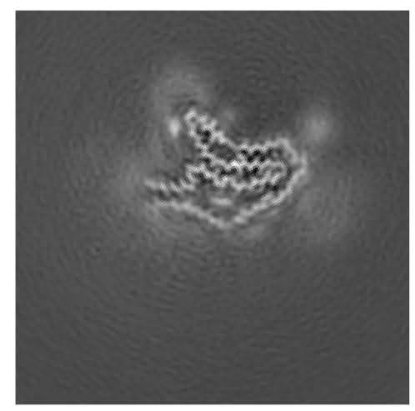

Case 3

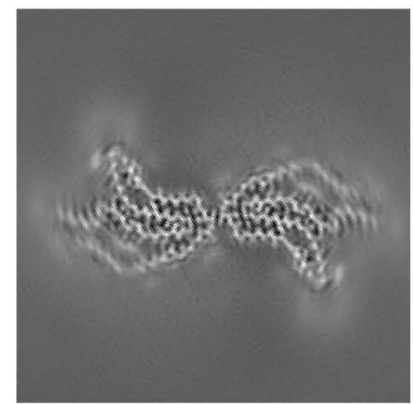

b

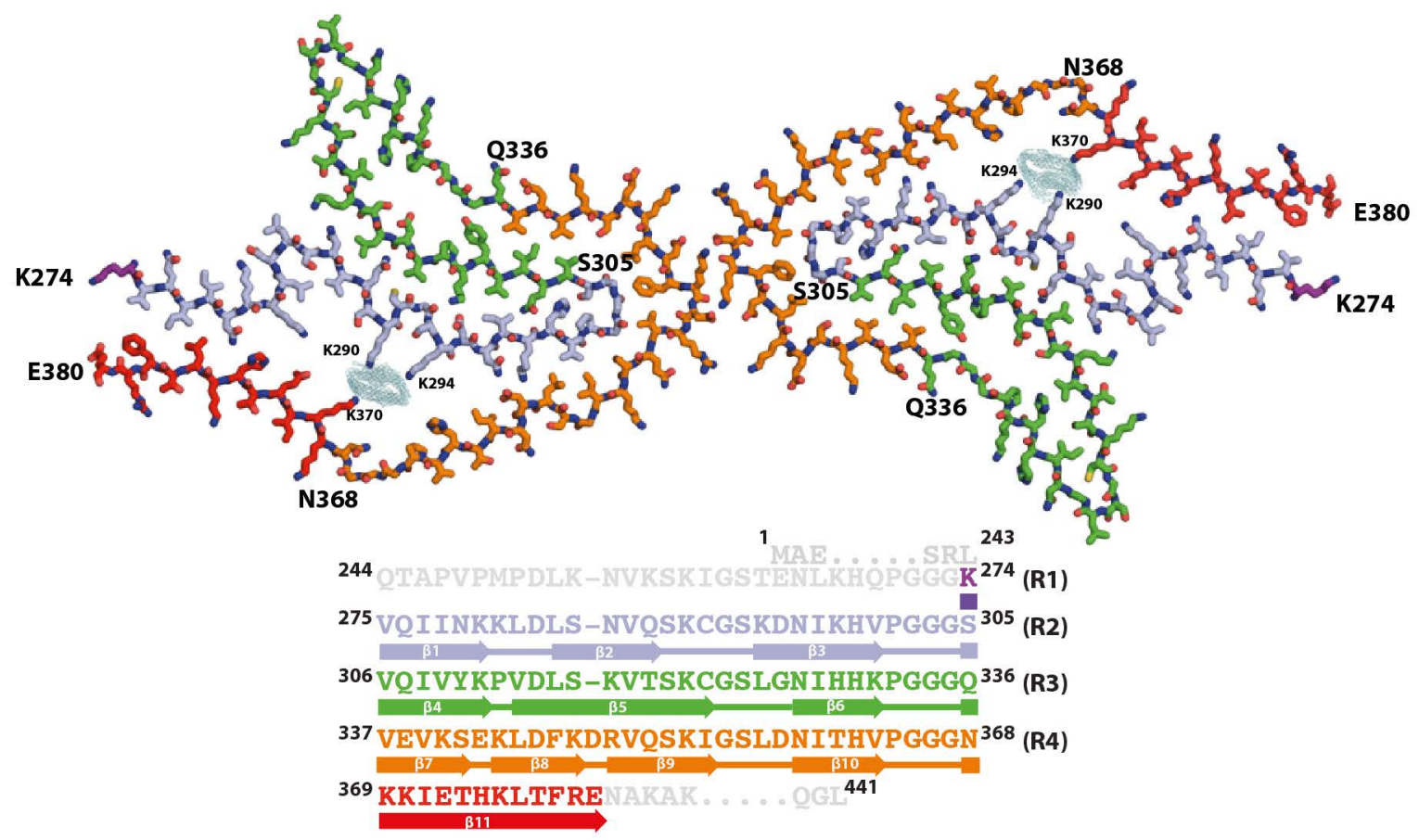


a

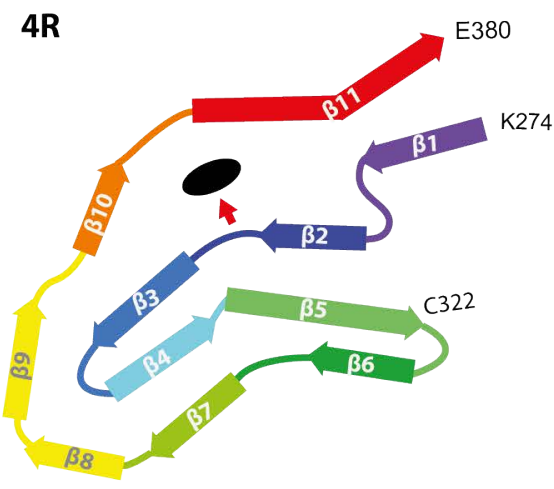

$$
\text { CBD fold }
$$

$$
3 R+4 R
$$

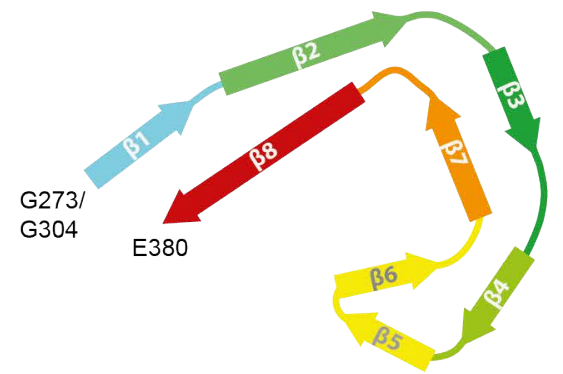

Alzheimer fold
3R

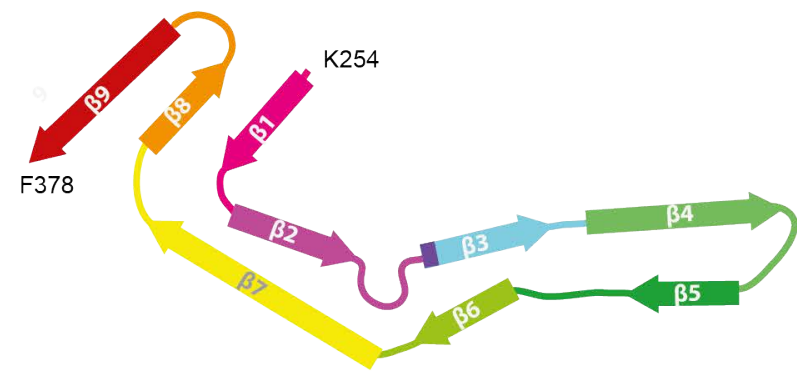

Pick fold

\section{$3 R+4 R$}

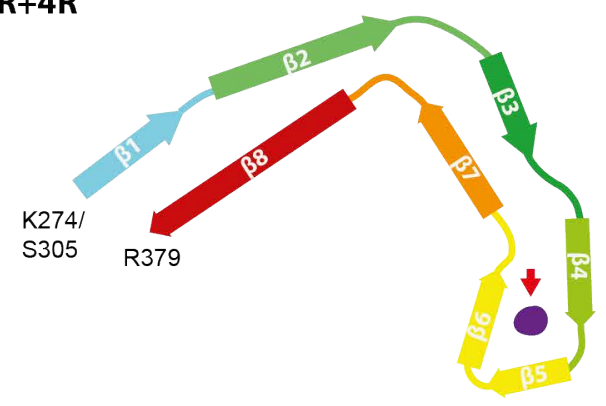

CTE fold

b

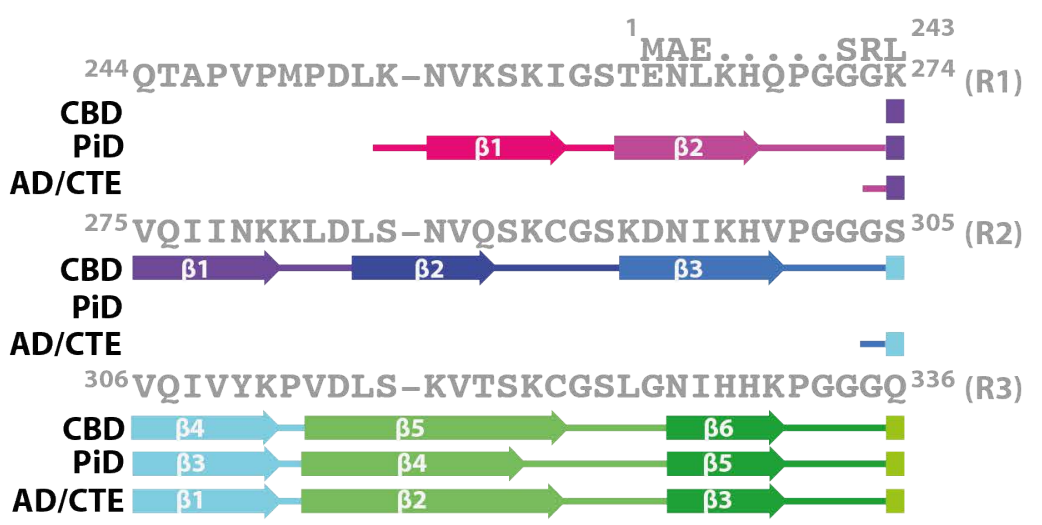

\footnotetext{
337 VEVKSERLDFRDRVQSKI GSLDNITHVP GGGN
CBD

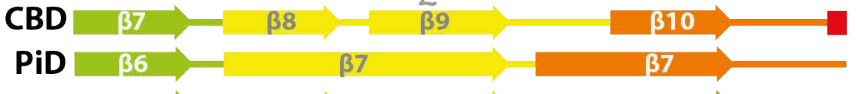

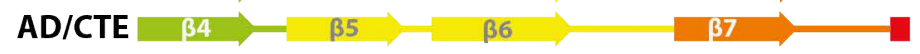

369 KKIETHKLTFRENAKAK . . . . QGL 441

CBD

PiD

B11 


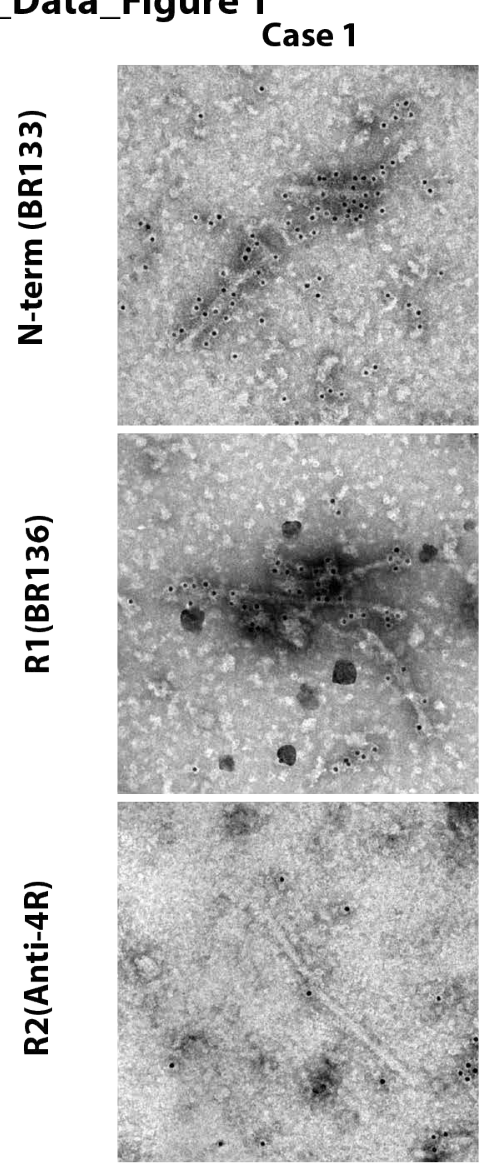

\section{Case 2}
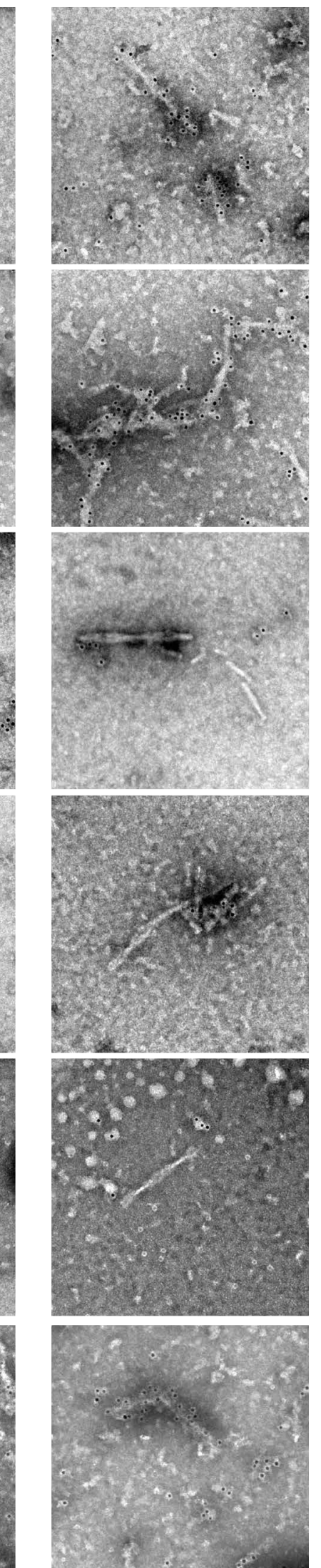

Case 3
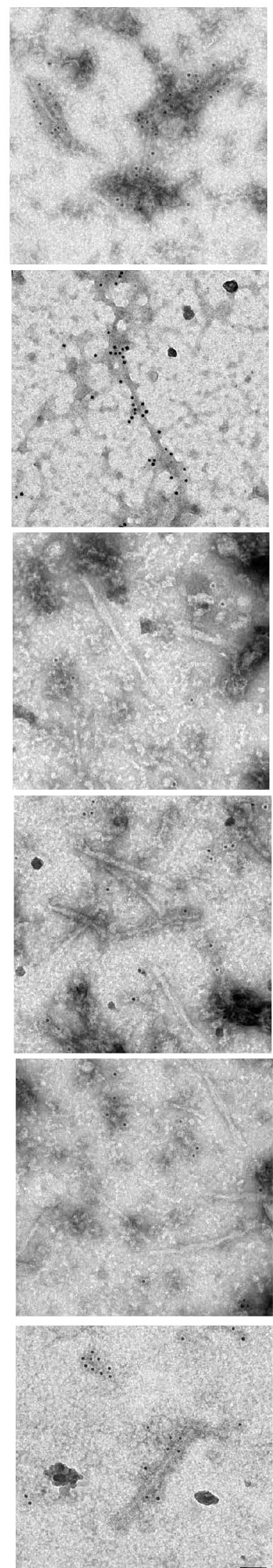
Extended_Data_Figure 2

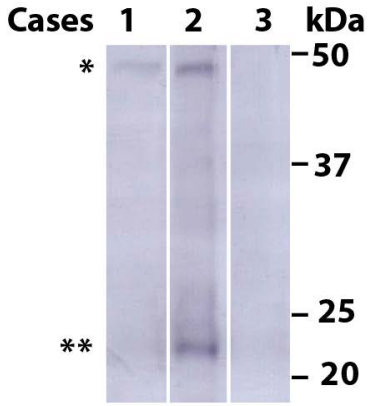


Extended_Data_Figure 3
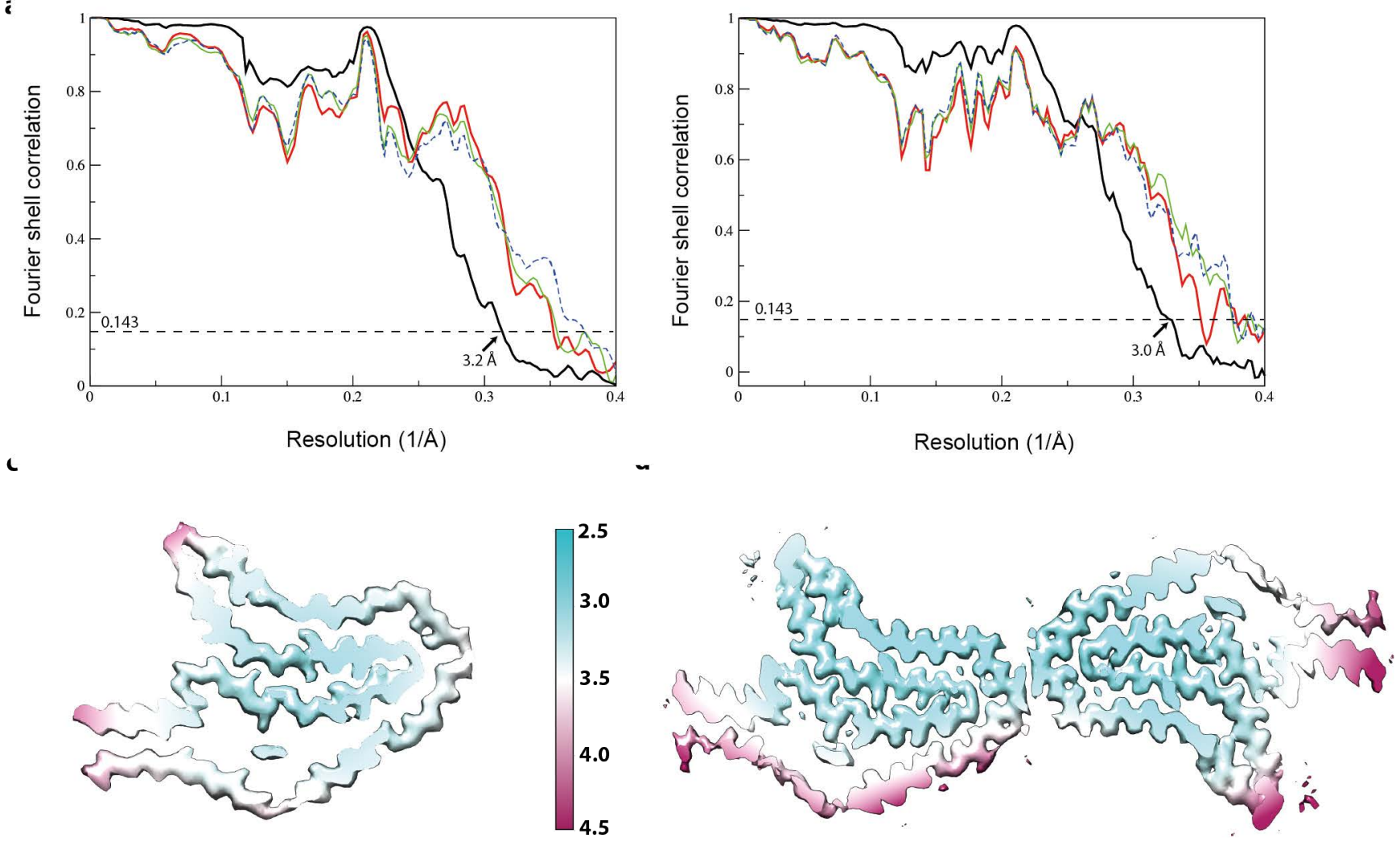

e

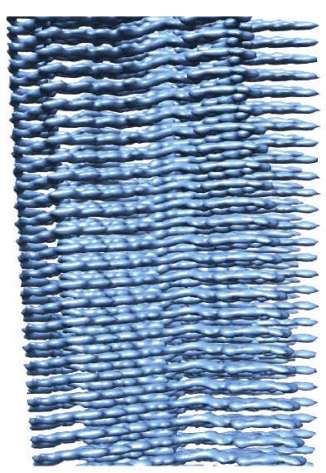

g
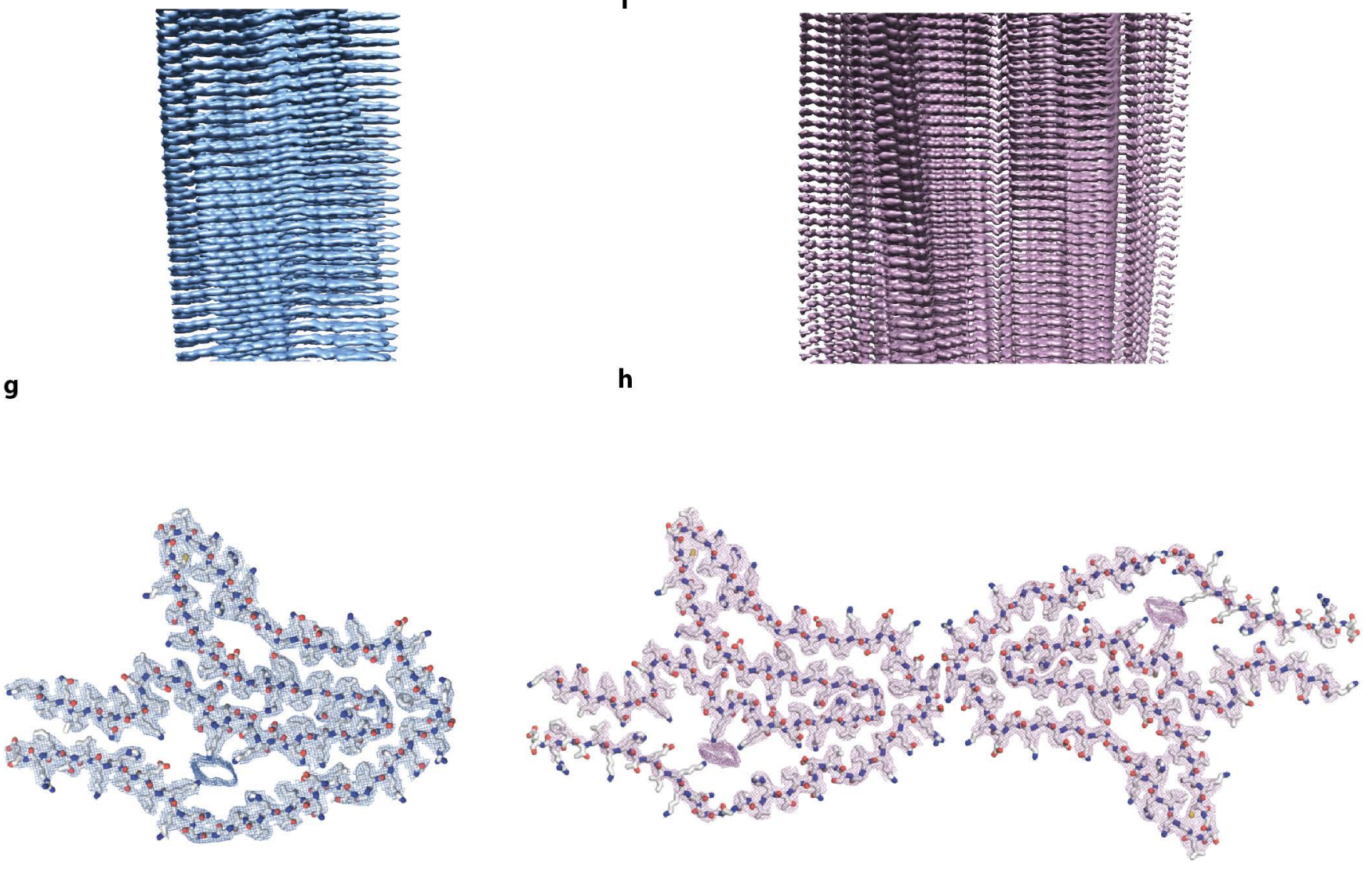


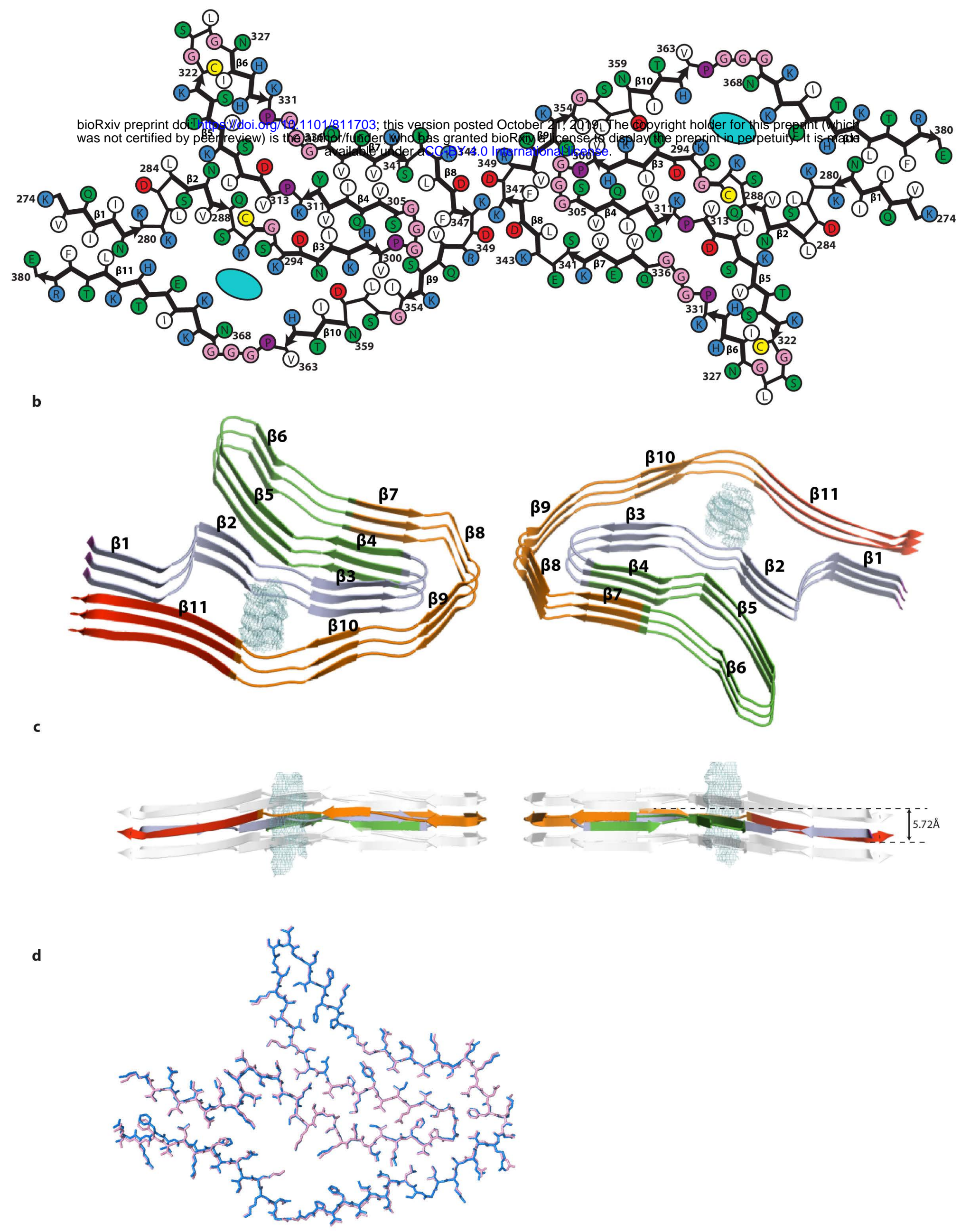


Extended_Data_Figure 5

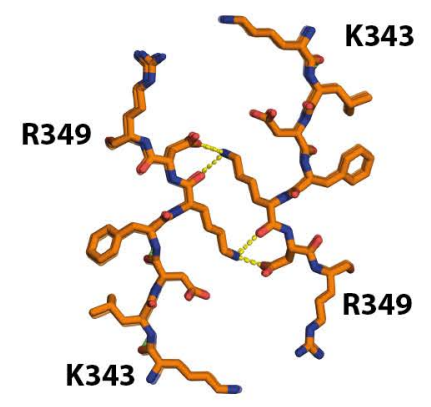

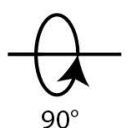

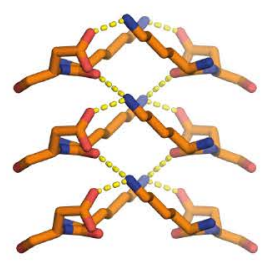

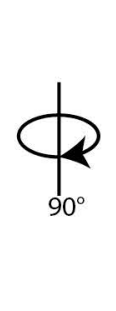

K343

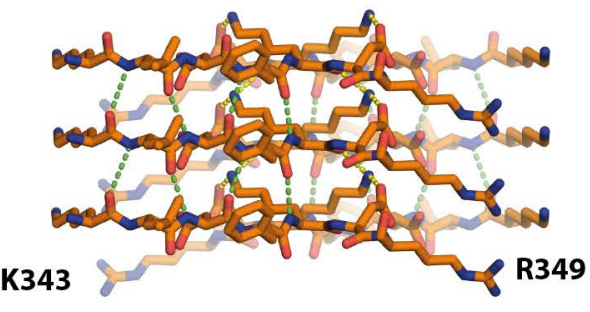




\section{Extended_Data_Figure 6}

a
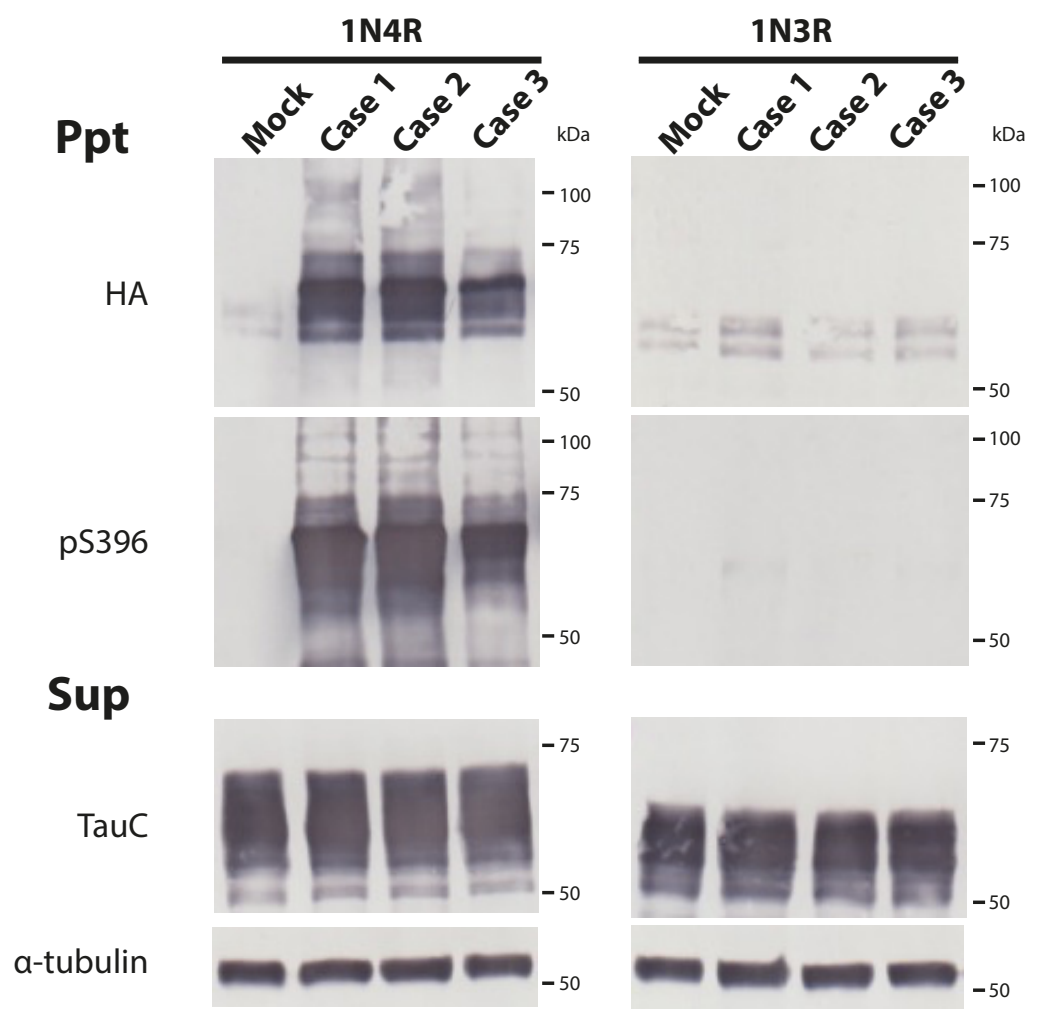

b

1N4R

1N3R
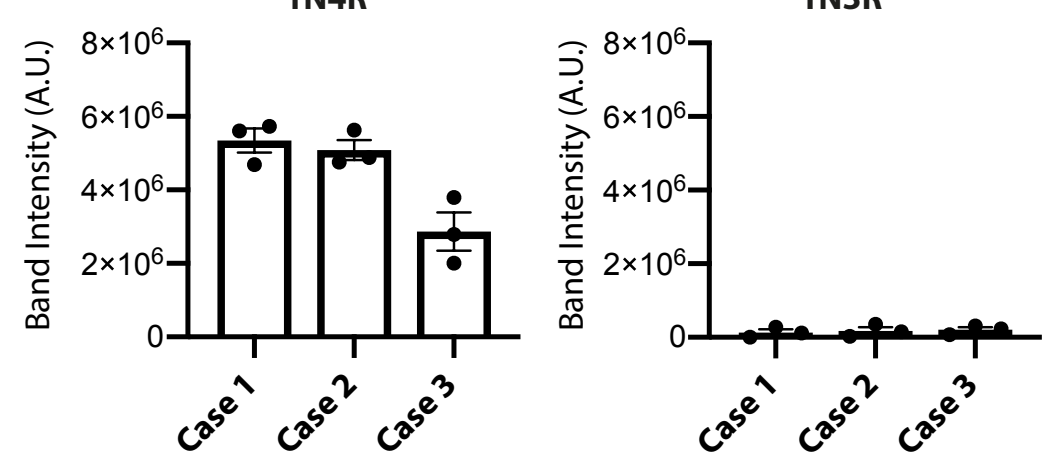
bioRxiv preprint doi: https://doi.org/10.1101/811703; this version posted October 21,2019 . The copyright holder for this preprint (which was not certified by peer review) is the author/funder, who has granted bioRxiv a license to display the preprint in perpetuity. It is made available under aCC-BY 4.0 International license.

Extended Data Table 1 Cryo-EM data collection, refinement and validation statistics

\begin{tabular}{|c|c|c|c|c|c|c|}
\hline & \multicolumn{2}{|c|}{ Case 1} & \multicolumn{2}{|c|}{ Case2 } & \multicolumn{2}{|c|}{ Case3 } \\
\hline & TypeI & TypeII & TypeI & TypeII & TypeI & TypeII \\
\hline \multicolumn{7}{|c|}{ Data collection and processing } \\
\hline Magnification & \multicolumn{2}{|c|}{$\mathrm{x} 130,000$} & \multicolumn{2}{|c|}{$\mathrm{x} 130,000$} & \multicolumn{2}{|c|}{$\mathrm{x} 130,000$} \\
\hline Voltage $(\mathrm{kV})$ & \multicolumn{2}{|c|}{300} & \multicolumn{2}{|c|}{300} & \multicolumn{2}{|c|}{300} \\
\hline $\begin{array}{l}\text { Electron exposure } \\
\left(\mathrm{e}^{-} / \AA^{2}\right)\end{array}$ & \multicolumn{2}{|c|}{53.84} & \multicolumn{2}{|c|}{$55-100.0$} & \multicolumn{2}{|c|}{52.5} \\
\hline Defocus range $(\mu \mathrm{m})$ & \multicolumn{2}{|c|}{-1.7 to -2.8} & \multicolumn{2}{|c|}{-1.7 to -2.8} & \multicolumn{2}{|c|}{-1.7 to -2.8} \\
\hline Pixel size $(\AA)$ & \multicolumn{2}{|c|}{1.15} & \multicolumn{2}{|c|}{1.15} & \multicolumn{2}{|c|}{1.15} \\
\hline $\begin{array}{l}\text { Initial particle } \\
\text { images (no.) }\end{array}$ & 135,646 & 129,812 & 188,074 & 137,069 & 198,035 & 632,918 \\
\hline $\begin{array}{l}\text { Final particle images } \\
\text { (no.) }\end{array}$ & 24,073 & 20,752 & 24,266 & 11,259 & 26,545 & 355,187 \\
\hline Map resolution $(\AA)$ & 3.2 & 3.0 & 5.0 & 3.5 & 3.8 & 3.0 \\
\hline Helical rise $(\AA)$ & -0.845 & -0.61 & -0.845 & -0.61 & -0.845 & -0.61 \\
\hline Helical twist $\left({ }^{\circ}\right)$ & 4.786 & 4.786 & 4.786 & 4.786 & 4.786 & 4.786 \\
\hline \multicolumn{7}{|l|}{ Refinement } \\
\hline $\begin{array}{l}\text { Map sharpening } B \\
\text { factor }\left(\AA^{2}\right)\end{array}$ & -26.63 & -25.75 & - & - & - & - \\
\hline \multicolumn{7}{|l|}{ Model composition } \\
\hline $\begin{array}{l}\text { Non-hydrogen } \\
\text { atoms }\end{array}$ & 2439 & 4878 & - & - & - & - \\
\hline Protein residues & 321 & 642 & - & - & - & - \\
\hline \multicolumn{7}{|l|}{ R.m.s. deviations } \\
\hline Bond lengths $(\AA)$ & 0.013 & 0.011 & - & - & - & - \\
\hline Bond angles $\left({ }^{\circ}\right)$ & 1.154 & 1.072 & - & - & - & - \\
\hline \multicolumn{7}{|l|}{ Validation } \\
\hline MolProbity score & 1.61 & 1.53 & - & - & - & - \\
\hline Clashscore & 2.2 & 4.11 & - & - & - & - \\
\hline Poor rotamers (\%) & 0.0 & 0.0 & - & - & - & - \\
\hline \multicolumn{7}{|l|}{ Ramachandran plot } \\
\hline Favored (\%) & 87.3 & 95.24 & - & - & - & - \\
\hline Allowed (\%) & 12.7 & 4.76 & - & - & - & - \\
\hline Disallowed (\%) & 0.0 & 0.0 & - & - & - & - \\
\hline \multicolumn{7}{|l|}{ EMDB } \\
\hline PDB & & & & & & \\
\hline
\end{tabular}

